\title{
MAGNETOOPTICAL PROPERTIES OF GaAs:Mn
}

\author{
J. Szczytko, A. Stachow, W. Mac, A. Twardowski \\ Institute of Experimental Physics, Warsaw University \\ Hoża 69, 00-681 Warsaw, Poland
}

P. BECLA

Massachusetts Institute of Technology, Cambridge, USA

AND J. TWORZYDEO

Institute of Theoretical Physics, Warsaw University, Hoża 69, 00-681 Warsaw, Poland

\begin{abstract}
Magnetoreflectance from GaAs:Mn was measured in the free exciton range for $B \leq 5 \mathrm{~T}$ and $T=2.0 \mathrm{~K}$. Combining optical results with magnetization data the value for the exchange parameter $N_{0} \alpha-N_{0} \beta=-(2.3 \pm 0.6) \mathrm{eV}$ was obtained. Assuming that $N_{0} \alpha \approx+0.2 \mathrm{eV}$, the ferromagnetic $p-d$ exchange interaction was found with the magnitude $N_{0} \beta \approx+2.5 \mathrm{eV}$.

PACS numbers: $78.20 . \mathrm{Ls}$
\end{abstract}

There are two basic issues which bring GaAs:Mn to be an interesting system. The first one is the problem of Mn dopant in III-V compounds, which has been studied for many years but the actual situation is not well understood yet [1-7]. The mostly accepted model assumes that the neutral $\mathrm{Mn}$ acceptor center is formed of $\mathrm{Mn}^{3+}$ core with tightly bound electron and a weakly bound hole $\left(\mathrm{Mn}^{2+}+\right.$ hole $)$ [1] (the so-called magnetic acceptor complex (MAC)). This model explains the results of EPR experiments but fails to describe magnetization and susceptibility data [4]. On the other hand, randomly distributed, localized magnetic moments of Mn ions classify GaAs:Mn to the group of the so-called diluted magnetic semiconductors (DMS) [7]. So far most of the research has been focused on II-VI DMS, whereas results for III-V compounds are very limited.

In this communication we report the results of exchange-induced band splitting of bulk GaAs heavily doped with Mn. This study was done by means of the free exciton magnetospectroscopy. Reflectance and the degree of polarization of reflectance in Faraday configuration were measured at $T=2.0 \mathrm{~K}$ and magnetic field $B \leq 5 \mathrm{~T}$ for several crystals with different $\mathrm{Mn}$ concentration (below $0.1 \%$ mole). Additionally magnetization was measured at the same temperature and the field range using a SQUID magnetometer. The $\mathrm{Ga}_{1-x} \mathrm{Mn}_{x}$ As crystals used in this work were grown by the Czochralski method. Single-phase crystals were obtained only for low Mn concentrations, $x<0.001$. We selected three crystals with 
the concentration $x=0.00047$ (sample \#1), 0.00027 (\#2) and 0.00022 (\#3) (the concentration was estimated from the magnetization of the sample, assuming that magnetization is well approximated by the Brillouin function for spin $S=5 / 2$ (see e.g. Ref. [8] for details)).

In Faraday configuration there are four exciton lines: two of them in circularity right $\left(\sigma^{+}\right)$(lines $A$ and $B$ ) and other two in circularity left $\left(\sigma^{-}\right)$polarized light (lines $C$ and $D$ ). The lines $A$ and $D$ are three times stronger than $B$ and $C$. The splitting of the lines $A$ and $D$ provides a direct measure of the difference between the exchange constants [7]

$$
E_{D}-E_{A}=\left(N_{0} \alpha-N_{0} \beta\right) x(-\langle S\rangle)=\left(N_{0} \alpha-N_{\beta}\right) \frac{m k}{\mu_{\mathrm{B}}} M,
$$

where average spin $\langle S\rangle$ is obtained from the macroscopic magnetization $M$ (per unit mass), parameter $k$ is equal to $1 / 2$ for spin-only magnetic moment (e.g. $\mathrm{Mn}^{2+}$ ). We note that the exact value of $x$ does not enter into the determination of the exchange constants.

Instead of recording individual $\sigma^{+}$and $\sigma^{-}$spectra, we measured directly the difference $D(E)=I\left(\sigma^{-}\right)-I\left(\sigma^{+}\right)$and the sum $R(E)=I\left(\sigma^{-}\right)+I\left(\sigma^{+}\right)$. The advantage of such procedure is that $D(E)$ spectrum provides an indication of the sign of exciton splitting. The polarization spectra clearly show that the exciton splits in a magnetic field in the way that its component visible in circularly right polarized light $\left(\sigma^{+}\right)$is blue-shifted in respect of the $\sigma^{-}$component (Fig. 1). Similar

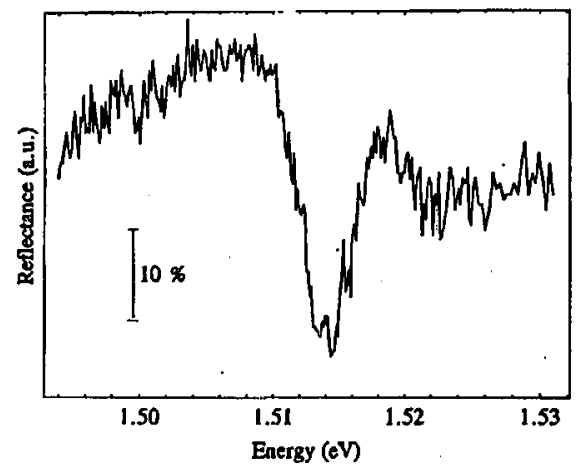

Fig. 1. The difference spectrum $D(E)=I\left(\sigma^{-}\right)-I\left(\sigma^{+}\right)$for GaAs:Mn (sample \#1) at $T=2.0 \mathrm{~K}$ and $B=5 \mathrm{~T}$. A dip in $D(E)$ spectrum indicates that $E\left(\sigma^{-}\right)<E\left(\sigma^{+}\right)$.

behavior was observed uniquely for Cr-based II-VI DMS [9] and is compatible with the ferromagnetic $p-d$ exchange. The opposite situation (antiferromagnetic $p-d$ exchange) was found for all $\mathrm{Mn}-, \mathrm{Fe}-$ and Co-based II-VI DMS [7].

From $D(E)$ and $R(E)$ the individual $I\left(\sigma^{-}\right)$and $I\left(\sigma^{+}\right)$spectra were obtained. Only one exciton line in each polarization was visible. The observed excitonic structures were ascribed to the exciton lines $A$ and $D$. Therefore $I\left(\sigma^{-}\right)-I\left(\sigma^{+}\right)$ splitting approximates $E_{D}-E_{A}$. Comparing the exciton splitting with magnetization we estimated the $s, p-d$ exchange parameter $N_{0} \alpha-N_{0} \beta=-2.0 \pm 0.6 \mathrm{eV}$ 


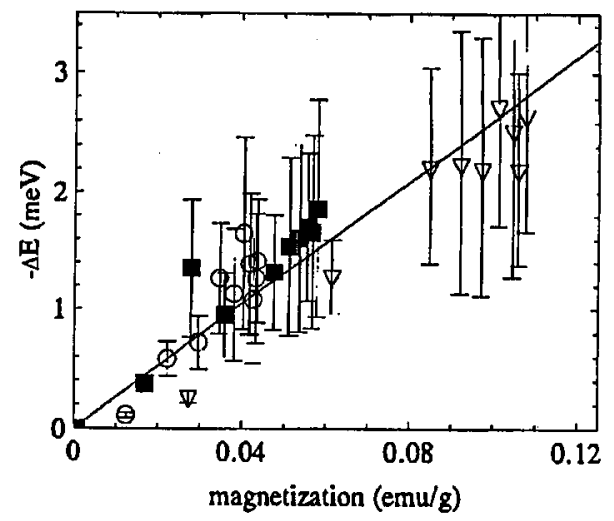

Fig. 2. The splitting of the free exciton versus magnetization for GaAs:Mn. The straight line corresponds to $N_{0} \alpha-N_{0} \beta=-2.0 \mathrm{eV}$.

(Fig. 2). Assuming that the measured exciton splitting $\Delta E$ is the weighted average of the strong and the weak lines splitting: $\Delta E=(3 / 4)\left(E_{A}-E_{D}\right)+(1 / 4)\left(E_{B}-E_{C}\right)$ (which results from the weak and the strong lines intensity ratio), and using $N_{0} \alpha \approx$ $+0.2 \mathrm{eV}$ (which is the case for II-VI DMS), we obtained $N_{0} \beta=+2.5 \pm 0.8 \mathrm{eV}$. The magnitude of $N_{0} \beta$ around $2 \mathrm{eV}$ is one of the largest for DMS [7]. We should however point out large experimental uncertainty of the present value, as well as the fact that it was obtained for the very diluted system. It is well known that for such case photocarriers may localize on magnetic ions (i.e. the nonperiodic potential wells resulting from substituting nonmagnetic cation by magnetic ion), which enhances the effect of $s, p-d$ interaction $[10,11]$. In this way the exciton splitting may largely deviate from the mean field and virtual crystal approximations result given by Eq. (1), eventually resulting in increase in $\Delta E / M$ with decreasing magnetic ion concentration. In other words the "exchange parameter" obtained from Eq. (1) may overestimate the real $\left|N_{0} \beta\right|$ value. It is not entirely certain whether this is the problem of the present paper since no appropriate calculations have been performed for GaAs:Mn and the accuracy of our data is too low to derive pertinent conclusions.

The obtained ferromagnetic sign of $p-d$ exchange can be hardly understood on the ground of $\mathrm{Mn}^{2+}\left(d^{5}\right)$ configuration. For the $\mathrm{Mn}^{2+}\left(d^{5}\right)$ ion in GaAs one may expect the energy level pattern of Mn $d$-levels similar to that for Mn-based II-VI DMS [7]. Such situation would yield antiferromagnetic $p-d$ exchange (negative $N_{0} \beta$ ), contrary to the present observation. On the other hand, the situation of $\mathrm{Mn}$ center in GaAs is different from the one in II-VI compounds, because Mn forms magnetic acceptor complex. The acceptor level located $0.113 \mathrm{eV}$ above the top of the valence band may substantially contribute to the exchange. To check this possibility we considered the virtual hole transfer from the acceptor level to the valence band and back (kinetic exchange). The calculations were done assuming that the $\mathrm{Mn}^{2+}$ bound hole wave function is of the same symmetry as for the top of the valence band. The obtained second-order energy corrections correspond to 
the ferromagnetic splitting of the electronic states of the valence band. However, the numerical estimation of this effect cannot be given yet, since the results are parameterized by unknown matrix element.

The observed $N_{0} \beta>0$ suggests that the ferromagnetic exchange dominates over conventional $p-d$ antiferromagnetic mechanism typical of Mn II-VI DMS.

\section{Acknowledgment}

This work was partially supported by the the Committee for Scientific Research (Poland) and National Science Foundation (USA).

\section{References}

[1] J. Schneider, U. Kaufmann, W. Wilkening, M. Baeumler, F. Kohl, Phys. Rev. Lett. 59, 240 (1987).

[2] R.A. Chapman, W.G. Hutchinson, Phys. Rev. Lett. 18, 443 (1967); L. Montelius, S. Nilsson, L. Samuelson, E. Janzen, M. Ahlstrom, J. Appl. Phys. 64, 1564 (1988).

[3] A. Petrou, M.C. Smith, C.H. Perry, J.M. Worlock, J. Warnock, R.L. Aggarwal, Solid State Commun. 55, 865 (1985).

[4] W. Mac, A. Twardowski, A.M. Hennel, in: Proc. 22 Int. Conf. on the Physics of Semiconductors, Vancouver 1994, Ed. D.J. Lockwood, World Scientific, Singapore 1995, p. 2569.

[5] X.C. Liu, D. Heiman, J. Hao, K.C. Hsieh, in: Proc. Int. Workshop on Diluted Magnetic Semiconductors, Linz (Austria) 1994, Eds. H. Heinrich, J.P. Mullin, Material Science Forum, Vol. 182-184, Trans Tech Publications, Switzerland 1995, p. 627.

[6] X.C. Liu, D. Heiman, J. Hao, K.C. Hsieh, in: Proc. 11 Int. Conf. on High Magnetic Fields in Semiconductor Physics, SEMIMAG-94, Ed. D. Heiman, World Scientific, Singapore 1995, p. 658.

[7] Semiconductors and Semimetals, Vol. 25, Diluted Magnetic Semiconductors, Eds. J.K. Furdyna, J. Kossut, Academic Press, New York 1988; Diluted Magnetic Semiconductors, Eds. M. Balkanski, M. Averous, Plenum Press, New York 1991; J. Kossut, W. Dobrowolski, in: Handbook of Magnetic Materials, Ed. K.H.J. Buschow, Vol. 7, North-Holland, Amsterdam 1993, p. 231.

[8] A. Twardowski, P. Glod, P. Wise, J.E. Crow, M. Demianiuk, Phys. Rev. B 46, 7537 (1992).

[9] W. Mac, Nguyen The Khoi, A. Twardowski, J.A. Gaj, M. Demianiuk, Phys. Rev. Lett. 71, 2327 (1993); A. Twardowski, in: Proc. Int. Workshop on Diluted Magnetic Semiconductors, Linz (Austria) 1994, Eds. H. Heinrich, J.P. Mullin, Material Science Forum, Vol. 182-184, Trans Tech Publications, Switzerland 1995, p. 599.

[10] C. Benoit à la Guillaume, D. Scalbert, T. Dietl, Phys. Rev. B 46, 9853 (1992); D. Scalbert, A. Ghazali, C. Benoit à la Guillaume, Phys. Rev. B 48, 17752 (1993).

[11] J. Tworzydło, Phys. Rev. B 50, 14591 (1994); J. Tworzydło, in: Proc. Int. Workshop on Diluted Magnetic Semiconductors, Linz (Austria) 1994, Eds. H. Heinrich, J.P. Mullin, Trans Tech Publications, Switzerland 1995, p. 593. 